\title{
The thermoelastic dynamic response of thick closed laminated shell
}

\author{
Ke Wei Ding \\ Department of Civil Engineering, Anhui Institute of Architecture and Industry, Hefei 230022, Anhui, P.R. China \\ Tel.: +86 551 3519737; Fax: +86 551 3517457; E-mail: dingkw@yahoo.com
}

Received 16 April 2004

Revised 29 June 2004

\begin{abstract}
Giving up any assumptions about displacement models and stress distribution, weak formulation of mixed state equations including boundary conditions of laminated cylindrical shell are presented. Thermal stresses mixed Hamilton equation of closed cylindrical shell is established. The analytical solutions are obtained for the thermoelastic dynamic response of a thick closed laminated shell subjected to temperature variation. Every equation of elasticity can be satisfied, and all elastic constants can be taken into account. Arbitrary precision of a desired order can be obtained.
\end{abstract}

Keywords: Laminated cylindrical shell, weak formulation, thermoelastic dynamic response, analytical solution

\section{Introduction}

The thermal stresses of cylindrical structure exposed to rapidly changing thermal environments can be of prime importance in the design of many industrial facilities. The thermoelastic analysis of cylindrical shells have received wide spread attention in recent years [1-7]. But most of theories are established on some hypotheses. For example, assume that the mechanical quantities are the polynomials of a certain coordinate variable. We have proved that the true solution for each mechanical quantity cannot be a polynomial of any coordinate variable [8]. If the form of a polynomial is adopted, the incompatibility among the fundamental equations must appear in the deductive process, and only some of elastic constants can be taken into account. This contradiction results in errors in all of the current theories, especially in thicker plates and shells. Analytical solution to three-dimensional elasticity theory are valuable not only in their own right, but also as useful benchmarks for verifying mathematical procedures leading to approximate solutions, and for providing exact structure design to achieve high performance structural objectives. This is even more important. Recently, three-dimensional static, dynamic, thermoelastic and buckling analysis of homogeneous and laminated composite cylinders have been studied by Soldatos and Ye [9]. Huang and Tauchert [10] examined the thermal stresses in double-curved cross-ply laminates. Thermal stresses in an axisymmetric double-layer annular circular cylinder with interlayer thermal resistance were analyzed by Chen and Lee [11]. Khdeir [12] investigated thermal deformations and stresses in cross-ply laminated circular cylindrical shells by means of state space approach. Ding and Tang [13,14] developed the method of state space, and gave the exact solution for axisymmetric vibration and buckling of laminated cylindrical shells having simply supported edge boundary and clamped edges, respectively. Exact thermoelastic solution for an axisymmetric problem of thick closed laminated shells has also been studied by Ding and Tang [15].

The analytical thermoelastic dynamic response analysis for the quite thick laminated cylindrical shell, to the author's knowledge, it is so difficult that few references have been found. In this paper, however, weak formulation of mixed state equations including boundary conditions of laminated cylindrical shell are presented, thermal stresses mixed Hamilton equation of closed cylindrical shell is established. Furthermore, for applying state space approach [16,17], the analytical solution is expressed for the thermoelastic dynamic response of the thick laminated closed cylindrical shells subjected to temperature variation. 


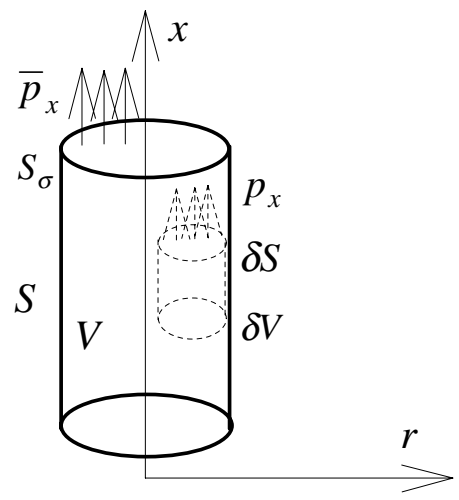

Fig. 1. The cylindrical shell.

\section{Weak formulation of mixed state equations}

A cylindrical shell is shown in Fig. 1. The principal elastic directions of the shell coincide with the coordinate axes. Let $u, v$ and $w$ be the displacement in the $x-, \theta-$ and $r$-directions, respectively. Also, let $V, S$ and $f_{i}$ be volume, boundary surface and body forces, respectively. $p_{x}, p_{\theta}$ and $p_{r}$ are the surface forces in the $x-, \theta-$ and $r$-directions, respectively. $\rho \ddot{u}$ is force of inertia, $\rho$ is the mass density. The equilibrium equation in the $x-$ direction for $\delta V$ can be written as

$$
\iint_{\delta S} p_{x} d s+\iiint_{\delta V} f_{x} d V-\iiint_{\delta V} \rho \ddot{u} d V=0
$$

$S_{\sigma}$ denote the portion of the edge boundary where tractions $\bar{p}_{x}$ is prescribed, for all shell we have

$$
\iint_{S} p_{x} d s+\iint_{S \sigma}\left(p_{x}-p_{x}\right) d s+\iiint_{V} f_{x} d V-\iiint_{V} \rho \ddot{u} d V=0
$$

By means of Green-formulation we can obtain

$$
\iiint_{V}\left(\begin{array}{c}
\partial \sigma_{x} \\
\partial x
\end{array}+\begin{array}{c}
1 \partial \tau_{x \theta} \theta \\
r \partial \theta
\end{array}+\begin{array}{c}
\partial \tau_{r x} \\
\partial r
\end{array}+\begin{array}{c}
\tau_{r x} \\
r
\end{array}+f_{x}-\rho \ddot{u}\right) d V+\iint_{S \sigma}\left(p_{x}-p_{x}\right) d s=0
$$

The equilibrium equation in the $\theta-$ direction for $\delta V$ can be written as

$$
\iint_{\delta S} p_{\theta} d s+\iiint_{\delta V} f_{\theta} d V-\iiint_{\delta V} \rho \ddot{v} d V=0
$$

for all shell we have

$$
\iint_{S} p_{\theta} d s+\iint_{S \sigma}\left(p_{\theta}-p_{\theta}\right) d s+\iiint_{V} f_{\theta} d V-\iiint_{V} \rho \ddot{v} d V=0
$$

By means of Green-formulation we can obtain

$$
\iiint_{V}\left(\begin{array}{c}
\partial \tau_{x \theta} \\
\partial x
\end{array}+\begin{array}{c}
1 \partial \sigma_{\theta} \\
r \partial \theta
\end{array}+\begin{array}{c}
\partial \tau_{r \theta} \\
\partial r
\end{array}+\begin{array}{c}
2 \tau_{r \theta} \\
r
\end{array}+f_{\theta}-\rho \ddot{v}\right) d V+\iint_{S \sigma}\left(p_{\theta}-p_{\theta}\right) d s=0
$$

the same as above, we have

$$
\iiint_{V}\left(\begin{array}{c}
\partial \tau_{r x} \\
\partial x
\end{array}+\begin{array}{c}
1 \partial \tau_{r \theta} \\
r \partial \theta
\end{array}+\begin{array}{c}
\partial \sigma_{r} \\
\partial r
\end{array}+\begin{array}{c}
\sigma_{r}-\sigma_{\theta} \\
r
\end{array}+f_{r}-\rho \ddot{w}\right) d V+\iint_{S \sigma}\left(p_{r}-p_{r}\right) d s=0
$$

In the light of definition of strain $\varepsilon_{x}$, we have

$$
\iiint_{\delta V} \varepsilon_{x} d x=\iiint_{\delta V} \partial u d x=\iint_{\delta S} u n_{x} d s
$$


Let $u$ is known displacement on $S_{u}$, for all shell we have

$$
\iiint_{V} \varepsilon_{x} d x=\iint_{S-S_{u}} u n_{x} d s+\iint_{S_{u}} u n_{x} d s=\iint_{S} u n_{x} d s+\iint_{S_{u}}(u-u) n_{x} d s
$$

By means of Green-formulation we can obtain

$$
\iiint_{V}\left(\begin{array}{l}
\partial u \\
\partial x
\end{array}-\varepsilon_{x}\right) d V+\iint_{S_{u}}(u-u) n_{x} d s=0
$$

the same as Eq. (2a), we have

$$
\begin{aligned}
& \iiint_{V}\left(\begin{array}{l}
\partial v \\
\partial \theta
\end{array}-\varepsilon_{\theta}\right) d V+\iint_{S_{u}}(v-v) n_{\theta} d s=0 \\
& \ldots \ldots \ldots \ldots \\
& \ldots \ldots \ldots \ldots \\
& \quad \iiint_{V}\left(\begin{array}{l}
1 \partial u \\
r \partial \theta
\end{array}+\frac{\partial v}{\partial x}-\gamma_{x \theta}\right) d V+\iint_{S_{u}}(u-u) n_{\theta} d s+\iint_{S_{u}}(v-v) n_{x} d s=0
\end{aligned}
$$

in which the usual index notation is used. The stress-strain relations of orthotropy is

$$
\{\sigma\}=[C]\left\{\varepsilon+\varepsilon_{T}\right\}
$$

where

$$
\begin{aligned}
& \{\sigma\}=\left[\begin{array}{llllll}
\sigma_{x} & \sigma_{\theta} & \sigma_{r} & \tau_{r \theta} & \tau_{r x} & \tau_{x \theta}
\end{array}\right]^{T}
\end{aligned}
$$

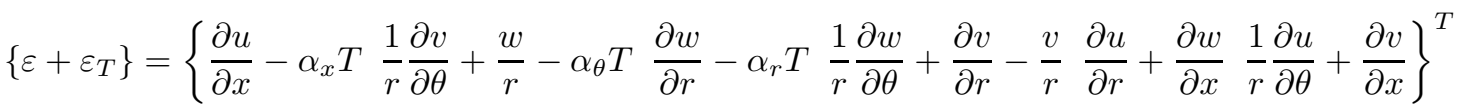

$\alpha_{x}, \alpha_{\theta}$ and $\alpha_{r}$ are the coefficients of thermal expansion, $T$ is the temperature rise from the stress-free state, the matrix [C] is the elastic stiffness matrix, for an orthotropic body, one has

$$
[C]=\left[\begin{array}{cccccc}
C_{11} & C_{12} & C_{13} & 0 & 0 & 0 \\
C_{12} & C_{22} & C_{23} & 0 & 0 & 0 \\
C_{13} & C_{23} & C_{33} & 0 & 0 & 0 \\
0 & 0 & 0 & C_{44} & 0 & 0 \\
0 & 0 & 0 & 0 & C_{55} & 0 \\
0 & 0 & 0 & 0 & 0 & C_{66}
\end{array}\right], \quad[S]=[C]^{-1}=\left[\begin{array}{cccccc}
S_{11} & S_{12} & S_{13} & 0 & 0 & 0 \\
S_{12} & S_{22} & S_{23} & 0 & 0 & 0 \\
S_{13} & S_{23} & S_{33} & 0 & 0 & 0 \\
0 & 0 & 0 & S_{44} & 0 & 0 \\
0 & 0 & 0 & 0 & S_{55} & 0 \\
0 & 0 & 0 & 0 & 0 & S_{66}
\end{array}\right]
$$

Substituting Eq. (3) into Eq. (2a)-(2f), then integrating by the weight function, i.e. multiply Eq. (1a)-(1c) by $\delta u, \delta v, \delta w$ and multiply Eq. (2a)-(2f) by $\delta \sigma_{x}, \ldots, \delta \tau_{x \theta}$, respectively. One denotes $q=\left(\begin{array}{l}u \\ v\end{array}\right)^{T}, p=$ $\left(\begin{array}{lll}\tau_{r x} & \tau_{r \theta} & \sigma_{r}\end{array}\right)^{T}, p_{1}=\left(\begin{array}{lll}\sigma_{x} & \sigma_{\theta} & \tau_{x \theta}\end{array}\right)^{T}, \boldsymbol{F}=\left(\begin{array}{ll}p & q\end{array}\right)^{T}$, weak formulation of mixed state equation including boundary conditions of cylindrical shell can be obtained

$$
\begin{aligned}
& \iiint_{V} \delta \boldsymbol{F} \cdot{ }_{\partial r}^{\partial} \boldsymbol{F} d V=\iiint_{V} \delta \boldsymbol{F} \cdot\left(\boldsymbol{H} F+\boldsymbol{T}_{F}+\boldsymbol{D}_{\mathbf{1}} \boldsymbol{p}_{\mathbf{1}}\right) d V+\iint_{S} \delta \boldsymbol{F} \cdot \boldsymbol{S}_{1} d s \\
& \iiint_{V} \delta \boldsymbol{p} \cdot\left(\boldsymbol{D}_{2} \boldsymbol{F}+\boldsymbol{T}_{p}+\boldsymbol{B} \boldsymbol{p}_{1}\right) d V+\iint_{S} \delta \boldsymbol{p} \cdot \boldsymbol{S}_{2} d s=0
\end{aligned}
$$

where $\left(\xi^{2}=\rho \partial^{2} / \partial t^{2}\right)$

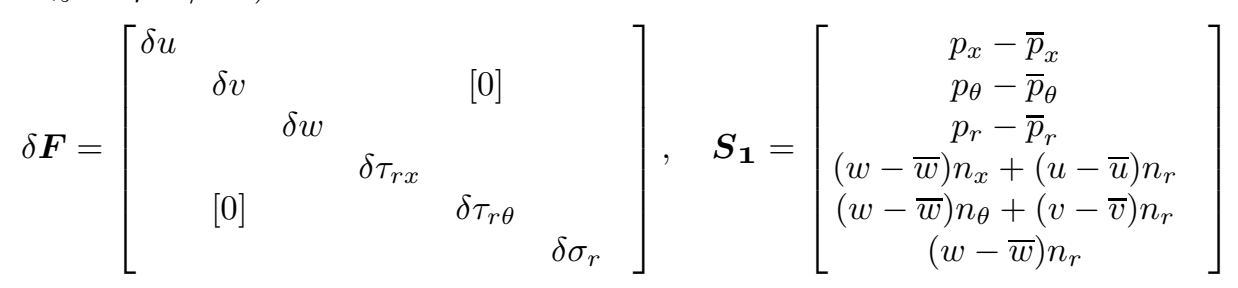




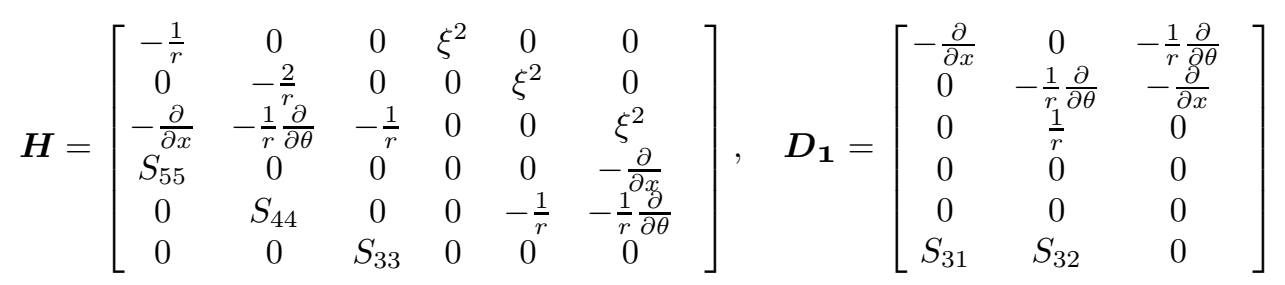

$$
\begin{aligned}
& \boldsymbol{S}_{2}=\left[\begin{array}{c}
(u-u) n_{x} \\
(v-v) n_{\theta} \\
(u-u) n_{\theta}+(v-v) n_{x}
\end{array}\right], \boldsymbol{B}=\left[\begin{array}{ccc}
S_{11} & S_{12} & 0 \\
S_{21} & S_{22} & 0 \\
0 & 0 & S_{66}
\end{array}\right], \delta \boldsymbol{p}=\left[\begin{array}{ccc}
\delta \sigma_{x} & 0 & 0 \\
0 & \delta \sigma_{\theta} & 0 \\
0 & 0 & \delta \tau_{x \theta}
\end{array}\right] \\
& \boldsymbol{D}_{2}=\left[\begin{array}{cccccc}
0 & 0 & S_{13} & -{ }_{\partial x}^{\partial} & 0 & 0 \\
0 & 0 & S_{23} & 0 & -{ }_{r}^{1} \partial & -{ }_{r}^{1} \\
0 & 0 & 0 & -{ }_{r}^{1} \partial \theta & -{ }_{\partial} \partial \theta & 0
\end{array}\right], \begin{array}{l}
\boldsymbol{T}_{F}=\left(\begin{array}{ccccccc}
-f_{x} & -f_{\theta} & -f_{r} & 0 & 0 & \alpha_{r} T
\end{array}\right)^{T} \\
\boldsymbol{T}_{p}=\left(\begin{array}{llllll}
\alpha_{x} T & \alpha_{\theta} T & 0 & 0 & 0 & 0
\end{array}\right)^{T}
\end{array}
\end{aligned}
$$

\section{Mixed state hamilton equation and its solution}

An orthotropic thick closed cylindrical shell is investigated, and selecting $(\zeta=m \pi / l)$

$$
\begin{aligned}
u & =\sum_{m} \sum_{n} u_{m n}(r) \cos \zeta x \cos (n \theta) e^{i \omega_{m n} t}, \quad \tau_{r x}=\sum_{m} \sum_{n} \tau_{r x, m n}(r) \cos \zeta x \cos (n \theta) e^{i \omega_{m n} t} \\
v & =\sum_{m} \sum_{n} v_{m n}(r) \sin \zeta x \sin (n \theta) e^{i \omega_{m n} t}, \quad \tau_{r \theta}=\sum_{m} \sum_{n} \tau_{r \theta, m n}(r) \sin \zeta x \sin (n \theta) e^{i \omega_{m n} t} \\
w & =\sum_{m} \sum_{n} w_{m n}(r) \sin \zeta x \cos (n \theta) e^{i \omega_{m n} t}, \quad \sigma_{r}=\sum_{m} \sum_{n} \sigma_{r, m n}(r) \sin \zeta x \cos (n \theta) e^{i \omega_{m n} t} \\
T & =\sum_{m} \sum_{n} T_{m n}(r) \sin \zeta x \cos (n \theta) e^{i \omega_{m n} t}
\end{aligned}
$$

Substituting Eqs (6) and (7) into Eqs (4) and (5), and letting

$$
\begin{gathered}
\omega=\omega_{m n}, C_{1}=-C_{13} / C_{33}, C_{2}=C_{11}-C_{13}^{2} / C_{33}, C_{3}=C_{12}-C_{13} C_{23} / C_{33} \\
C_{4}=C_{22}-C_{23}^{2} / C_{33}, C_{5}=-C_{23} / C_{33}, C_{7}=1 / C_{33}, C_{8}=1 / C_{55}, C_{9}=1 / C_{44} \\
C_{6}=C_{66}, C_{a}=C_{3} \alpha_{x}+C_{4} \alpha_{\theta}, C_{b}=C_{2} \alpha_{x}+C_{3} \alpha_{\theta}, C_{d}=C_{1} \alpha_{x}+C_{5} \alpha_{\theta}-\alpha_{r}
\end{gathered}
$$

Then we obtain the mixed state Hamilton equation of thermal stresses for the cylindrical shell for each combination of $m$ and $n$

$$
{ }_{d r}^{d} \boldsymbol{F}(r)=\boldsymbol{M}(r) \boldsymbol{F}(r)+\boldsymbol{B}(r)
$$

where

$$
\begin{aligned}
& \boldsymbol{F}(r)=\left[r u_{m n}(r) r v_{m n}(r) r w_{m n}(r) \tau_{r x, m n}(r) \tau_{r \theta, m n}(r) \sigma_{r, m n}(r)\right]^{T} \\
& \boldsymbol{M}(r)=\left[\begin{array}{cc}
\boldsymbol{A}^{T} & \boldsymbol{D} \\
\boldsymbol{E} & -\boldsymbol{A}
\end{array}\right]
\end{aligned}
$$




$$
\begin{aligned}
& \boldsymbol{A}^{T}=\left[\begin{array}{ccc}
1 & 0 & -\zeta \\
r & 2 & n \\
0 & C_{5}^{r} n & C_{5}+1
\end{array}\right], \quad-\boldsymbol{A}=\left[\begin{array}{ccc}
-{ }_{r} & 0 & C_{1} \zeta \\
0 & -{ }^{2} & -{ }_{5} n \\
-C_{1} \zeta & r & r
\end{array}\right]
\end{aligned}
$$

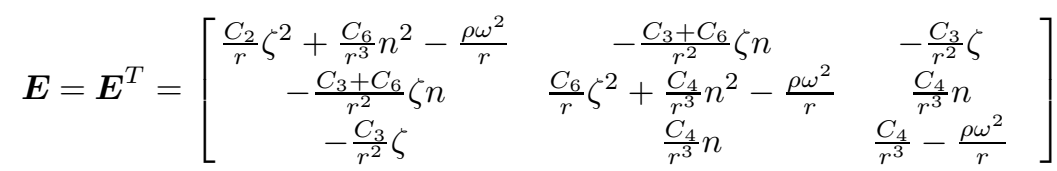

$$
\begin{aligned}
& \boldsymbol{D}=\boldsymbol{D}^{T}=\left[\begin{array}{ccc}
C_{8} r & 0 & 0 \\
0 & C_{9} r & 0 \\
0 & 0 & C_{7} r
\end{array}\right]
\end{aligned}
$$

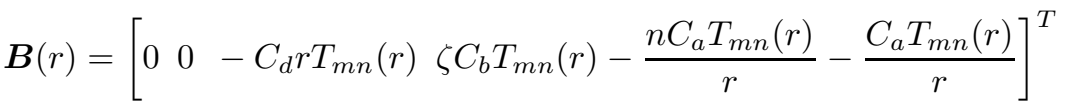

In order to solve Eq. (8), thick shell should be divided into some thin plies. If we find, from calculation, that the needful effective digits hardly change, it can be said that the results obtained with certain thin plies are exact within the prescribed accuracy limits. For the first ply, the solution of Eq. (8) is

$$
\boldsymbol{F}(r)=\boldsymbol{G}(r-a) \boldsymbol{F}(a)+\boldsymbol{C}(r-a)
$$

in which

$$
\boldsymbol{G}(r-a)=\exp [\boldsymbol{M} \cdot(r-a)], \boldsymbol{C}(r-a)=\int_{r} \exp [\boldsymbol{M} \cdot(r-\tau)] \boldsymbol{B}(\tau) d \tau
$$

Equation (9) is Hamiltonian matrix. The present result is exactly analogous to the Hamiltonian mechanics for a dynamic system. In order to calculate the matrix function $\boldsymbol{G}(r-a), \boldsymbol{C}(r-a)$, the eigenvalues of the matrix $\boldsymbol{M}$ must be considered. Let $\lambda_{1}, \lambda_{2}, \cdots, \lambda_{6}$ be the eigenvalues of the matrix $\boldsymbol{M}$ and $\boldsymbol{J}_{1}, \boldsymbol{J}_{2}, \cdots, \boldsymbol{J}_{6}$ are the eigenvectors, respectively. We know, from linear algebra, that there must be a matrix $\boldsymbol{R}=\left[\boldsymbol{J}_{1}, \boldsymbol{J}_{1}, \cdots, \boldsymbol{J}_{6}\right]$ and its inverse matrix $\boldsymbol{R}^{-1}$, which can change $\boldsymbol{M}$ into a diagonal matrix, and has

$$
\begin{aligned}
\boldsymbol{G}(r-a) & =\exp [\boldsymbol{M} \cdot(r-a)] \\
& =\boldsymbol{R} \cdot\left[\begin{array}{cccc}
e^{\lambda_{1}(r-a)} & & & 0 \\
& e^{\lambda_{2}(r-a)} & & \\
& & \ddots & \\
0 & & & e^{\lambda_{61}(r-a)}
\end{array}\right] \cdot \boldsymbol{R}^{-1}
\end{aligned}
$$

Therefore

$$
\boldsymbol{C}(r-a)=\int_{a}^{r} \boldsymbol{R} \cdot\left[\begin{array}{cccc}
e^{\lambda_{1}(r-\tau)} & & & 0 \\
& e^{\lambda_{2}(r-\tau)} & & \\
& & \ddots & \\
0 & & & e^{\lambda_{6}(r-\tau)}
\end{array}\right] \cdot \boldsymbol{R}^{-1} \boldsymbol{B}(\tau) d \tau
$$

In order to calculate multiple shell, we can apply transfer matrix method, for the first ply we have $\left(h_{1}\right.$-thickness of first ply)

$$
\boldsymbol{F}\left(r_{1}\right)=\boldsymbol{G}\left(-h_{1}\right) \boldsymbol{F}(a)+\boldsymbol{C}\left(-h_{1}\right)
$$

By virtue of the continuity conditions for displacements and stresses between the first and second ply, there must be

$$
\boldsymbol{F}\left(r_{2}\right)=\boldsymbol{G}\left(-h_{2}\right) \boldsymbol{G}\left(-h_{1}\right) \boldsymbol{F}(a)+\boldsymbol{G}\left(-h_{2}\right) \boldsymbol{C}\left(-h_{1}\right)+\boldsymbol{C}\left(-h_{2}\right)
$$




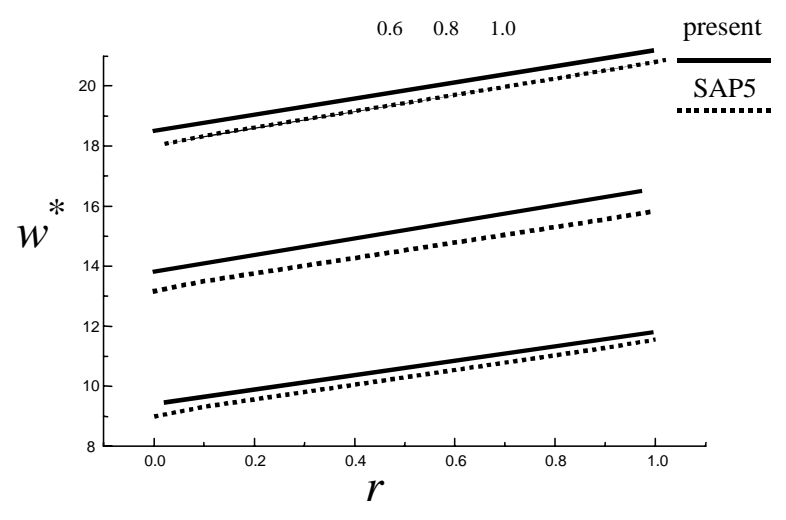

(a)

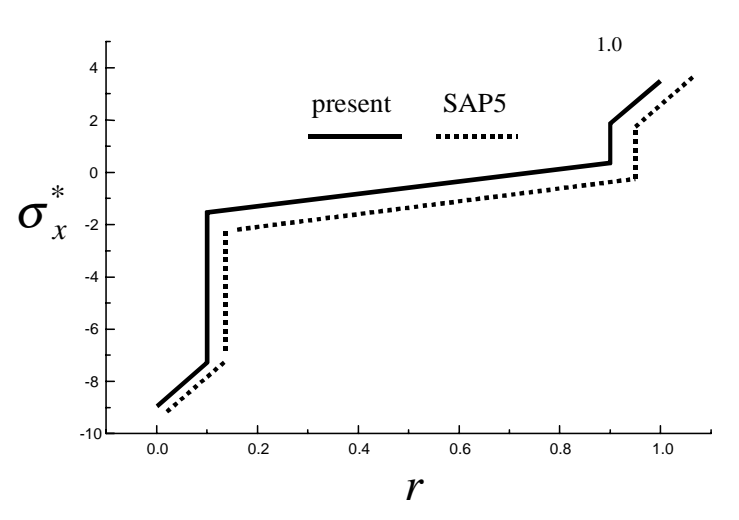

(c)

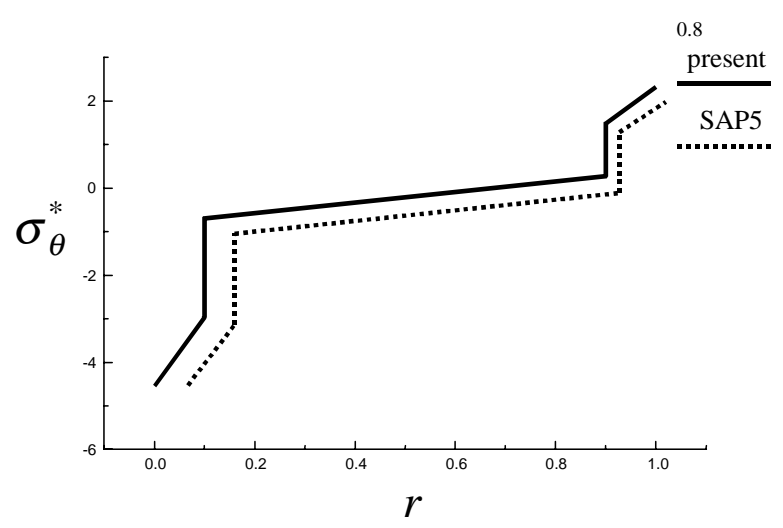

(b)

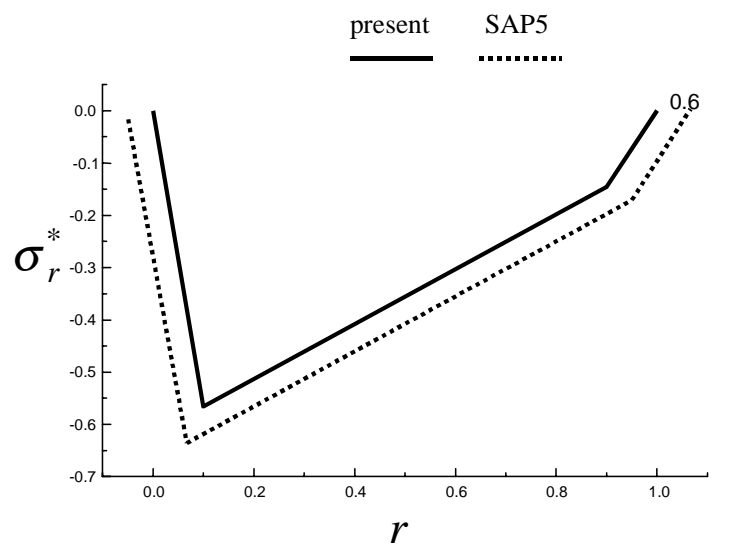

(d)

Fig. 2. Thermoelastic response of a three-ply shell.

On the analogy of this, the mechanical quantities of the interior surface and outer surfaces for the entire laminated shell can be linked together to be of the form ( $k$-the number of layers):

$$
\boldsymbol{F}(b)=\Pi \boldsymbol{F}(a)+\Pi
$$

in which

$$
\begin{aligned}
& \Pi=\boldsymbol{G}\left(-h_{k}\right) \boldsymbol{G}\left(-h_{k-1}\right) \boldsymbol{G}\left(-h_{k-2}\right) \cdots \boldsymbol{G}\left(-h_{2}\right) \boldsymbol{G}\left(-h_{1}\right) \\
& \Pi=\boldsymbol{G}\left(-h_{k}\right) \sum_{j=1}^{k-1}\left[\prod_{i=k-1}^{j+1} \boldsymbol{G}\left(-h_{i}\right) \boldsymbol{C}\left(-h_{j}\right)\right]+\boldsymbol{C}\left(-h_{k}\right)
\end{aligned}
$$

Actually, Eq. (13) is a matrix equation for six displacements of the outer and interior surfaces of a shell. $\Pi$ is a $(6 \times 6)$ constant matrix. $\Pi$ is a $(6 \times 1)$ column matrix. In the calculation of the thermoelastic dynamic response, considering boundary condition of interior and outer surfaces of shell, one has

$$
\sigma_{r, m n}(a)=\tau_{r x, m n}(a)=\tau_{r \theta, m n}(a)=\sigma_{r, m n}(b)=\tau_{r x, m n}(b)=\tau_{r \theta, m n}(b)=0
$$




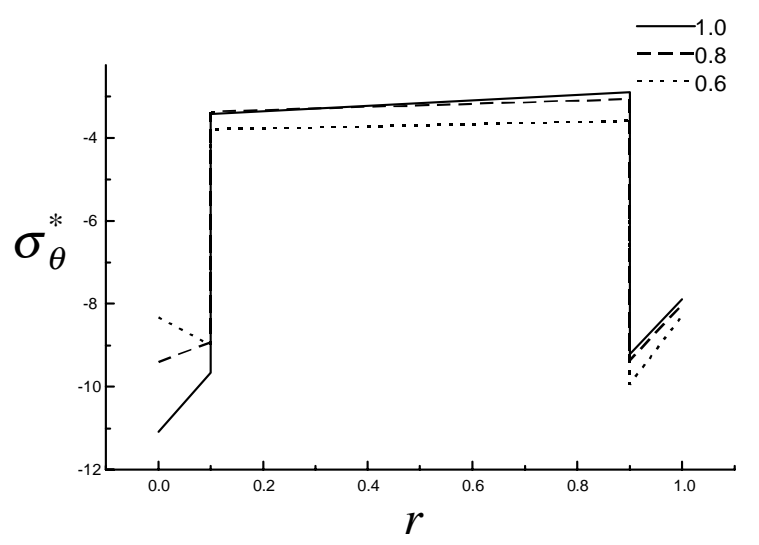

(a)

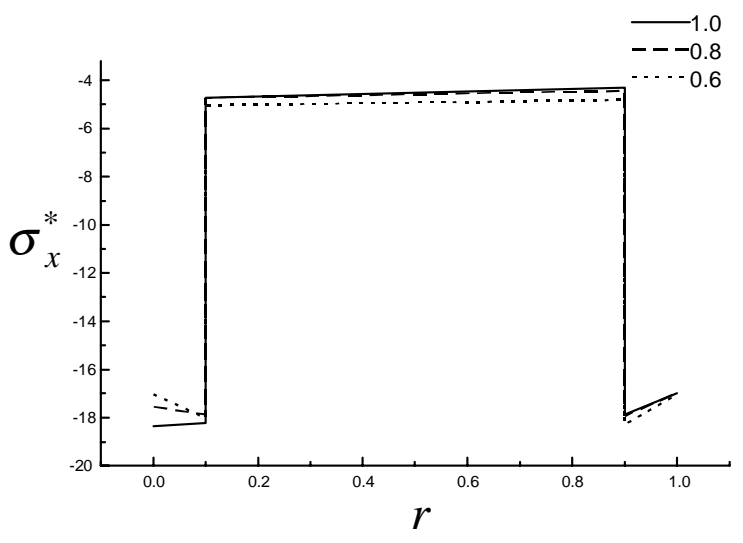

(b)

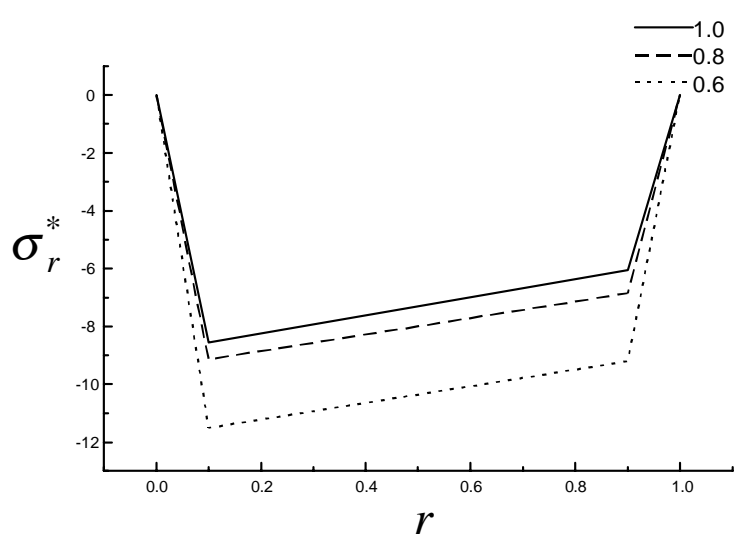

(c)

Fig. 3. Thermoelastic dynamic response of a three-ply laminated shell.

Selecting of the fourth, fifth and sixth rows of matrix Eq. (13) gives

$$
\left\{\begin{array}{l}
a u_{m n}(a) \\
a v_{m n}(a) \\
a w_{m n}(a)
\end{array}\right\}=-\left[\begin{array}{lll}
\Pi_{41} & \Pi_{42} & \Pi_{43} \\
\Pi_{51} & \Pi_{52} & \Pi_{53} \\
\Pi_{61} & \Pi_{62} & \Pi_{63}
\end{array}\right]^{-1} \cdot\left\{\begin{array}{l}
\Pi_{4} \\
\Pi_{5} \\
\Pi_{6}
\end{array}\right\}
$$

$u_{m n}(a), v_{m n}(a)$ and $w_{m n}(a)$ can be determined by Eq. (16). After finding these quantities, Eq. (14) is employed, other unknowns coefficients can be solved. After the unknowns are determined, the $\boldsymbol{F}(a)$ can be found by using Eq. (16) and the entire shell can be solved.

\section{Numerical example}

Example 1. Consider the thermoelastic response of a 3-plied laminated shell to the temperature variation $T=$ $T_{1} \sin \underset{l}{\pi x} \cos \theta$. The materials for the first and third layers are identical. Each layer has the same thermoelastic constants: 


$$
\begin{aligned}
& C_{12} / C_{11}=0.246269 \quad C_{13} / C_{11}=0.0831715 \quad C_{22} / C_{11}=0.543103 \\
& C_{23} / C_{11}=0.115017 \quad C_{33} / C_{11}=0.530172 \quad C_{44} / C_{11}=0.266810 \\
& C_{55} / C_{11}=0.159914, C_{66} / C_{11}=0.262931, C_{11}^{(1)} / C_{11}^{(2)}=5, \alpha_{x}=\alpha_{r}=3.0 \sigma_{\theta}
\end{aligned}
$$

where $C_{11}^{(1)}$ and $C_{11}^{(2)}$ denote $C_{11}$ of the materials corresponding to the first and second layer, respectively. The densities for the outer and middle layers are denoted by $\rho_{1}$ and $\rho_{2}\left(\rho_{1}=3, \rho_{2}=3\right)$, respectively. The laminated shell has the following geometry parameters:

$$
h_{1}=h_{3}=0.1 h \quad h_{2}=0.8 h \quad l=s=2 \pi R_{\circ}
$$

where $l=$ the length of the shell, $s=$ the arc length of middle surface and $R_{\circ}=$ the radius of middle surface. Some numerical results are obtained and we shown in Fig. 2(a-d) the variations of the displacement $\left(w^{*}=w C_{11}^{(2)} /\left(T_{1} \alpha_{\theta} h\right)\right)$ and stresses $\left(\sigma_{x}^{*}=\sigma_{x} /\left(T_{1} \alpha_{\theta}\right), \sigma_{\theta}^{*}=\sigma_{\theta} /\left(T_{1} \alpha_{\theta}\right), \quad \sigma_{r}^{*}=\sigma_{r} /\left(T_{1} \alpha_{\theta}\right)\right)$ through the thickness at $x=l / 2, \quad \theta=0$ of thick laminated shells with different ratios $h / R_{0}=0.6,0.8$ and 1.0, respectively. The results for three-dimensional finite element method (FEM) using SAP5 (Structural Analysis Program 5) are also given in Fig. 2. Because of the symmetry, 128 three-dimensional isoparametric elements (for 1/2 shell) with 20 nodes are employed in calculation. The average errors of present results and those of SAP5 are $3.67 \%$.

Example 2. Consider the thermpelastic dynamic response of above shell to the temperature variation $T=$ $T_{1} \sin \underset{l}{\pi x} \cos \theta e^{i 2 \pi t}$. Maximum values of $\sigma_{x}^{*}, \sigma_{\theta}^{*}, \sigma_{r}^{*}$ distributions in the thickness direction are shown in Fig. 3(a-c).

\section{Conclusion}

The analytical solution for the thermoelastic dynamic response of thick laminated closed cylindrical shells is investigated. The average errors of present results and those of SAP5 are 3.67\%. The principle and method suggested here have clear physical concepts and overcome the contradictions and limitations that arise from incompatibility among the fundamental equations in various theories of plates and shells. Numerical results denote that the methods of dividing the layer into several thin plies has the characteristics of fast convergence rate, satisfactory precision, and controlled error. The present study satisfies the continuity conditions of stresses and displacements at the interfaces. Solutions and method such as this have value for designing laminated composite structures in naval, aerospace and other engineering applications.

\section{Acknowledgment}

This project has been supported by the Natural Science Foundation of Anhui Province, P.R. China (2004kj090zd, 050440506). The author would like to express thanks to referees for their valuable comments.

\section{References}

[1] Y.C. Yang and C.K. Chen, Thermoelastic transient response of an infinitely long annular cylinder composed of two different materials, International Journal of Engineering Science 24 (1986), 569-581.

[2] E. Pardo and G. Sanchez Sarmiento, Analytical solution for unsteady thermal stress in an infinite cylinder composed of two materials, Journal of Thermal Stresses 10 (1987), 29-43.

[3] Mahesh gundappa and J.R. Thomas, analysis of thermal stresses in a solid circular cylinder subjected to conductive heat transfer with thermal boundary conductance, Journal of Thermal Stresses 8 (1985), 265-276.

[4] F.G. Yuan, Thermal stresses in thick laminated composite shells, Composite Structures 26 (1993), 63-75.

[5] R.B. Hetnarski and J. Ignaczak, Generalized thermoelasticity: closed-form solutions, Journal of Thermal Stresses 16(4) (1993), 473.

[6] K.K. Tamma and R.R. Namburu, Computational approaches with applications to non- classical and classical thermomechanical problems, Applied Mechanics and Review 50(9) (1997), 514-551.

[7] A.K. Noor and W.S. Burton, Computational models for sandwich panels and shells, Applied Mechanics and Review 49(3) (1996), 155-199. 
[8] K.W. Ding, Weak Formulation of Mixed State Equation and Boundary Value Problem of Theory of Elasticity, Ph.D. Dissertation (in Chinese), Dalian, Dalian University of Technology, 1998.

[9] K.P. Soldatos and J.Q. Ye, Three-dimensional Static, dynamic, thermoelastic and buckling analysis of homogeneous and laminated composite cylinders, Composite Structures 29 (1994), 131-143.

[10] N.N. Huang and T.R. Tauchert, Thermal stresses in doubly-curved cross-ply laminates, International Journal of Solids and Structures 29 (1992), 991-1000.

[11] W.C. Chen and W.H. Liu, Thermal buckling of antisymmetric angle-ply laminated plates an analytical levy-type solution, Journal of Thermal Stresses 16 (1993), 401-419.

[12] A.A. Khdeir, Thermoelastic analysis of cross-ply laminated circular cylindrical shells, International Journal of Solids and Structures 33(27) (1996), 4007-4017.

[13] K.W. Ding and L.M. Tang, Exact analysis for axisymmetric vibration and buckling of the thick laminated closed cylindrical shells in a Hamilton system, Journal of Sound and Vibration 206(3) (1997), 435-441.

[14] K.W. Ding and L.M. Tangin, Three-dimensional free vibration of thick laminated cylindrical shells with clamped edges, Journal of Sound and Vibration 220(1) (1999), 171-177.

[15] K.W. Ding and L.M. Tang, Exact thermoelastic solution for axisymmetric problem of thick closed laminated shells, Journal of Thermal Stresses 21(7) (1998), 751-761.

[16] A.A. Khdeir, A remark on the state-space concept applied to bending, buckling and free vibration of composite laminates, Computer and Structures 59(5) (1996), 813-817.

[17] K.W. Ding and L.M. Tang, Exact solution for axisymmetric thick laminated shells, Composite Structures 46(2) (1999), 125-129. 

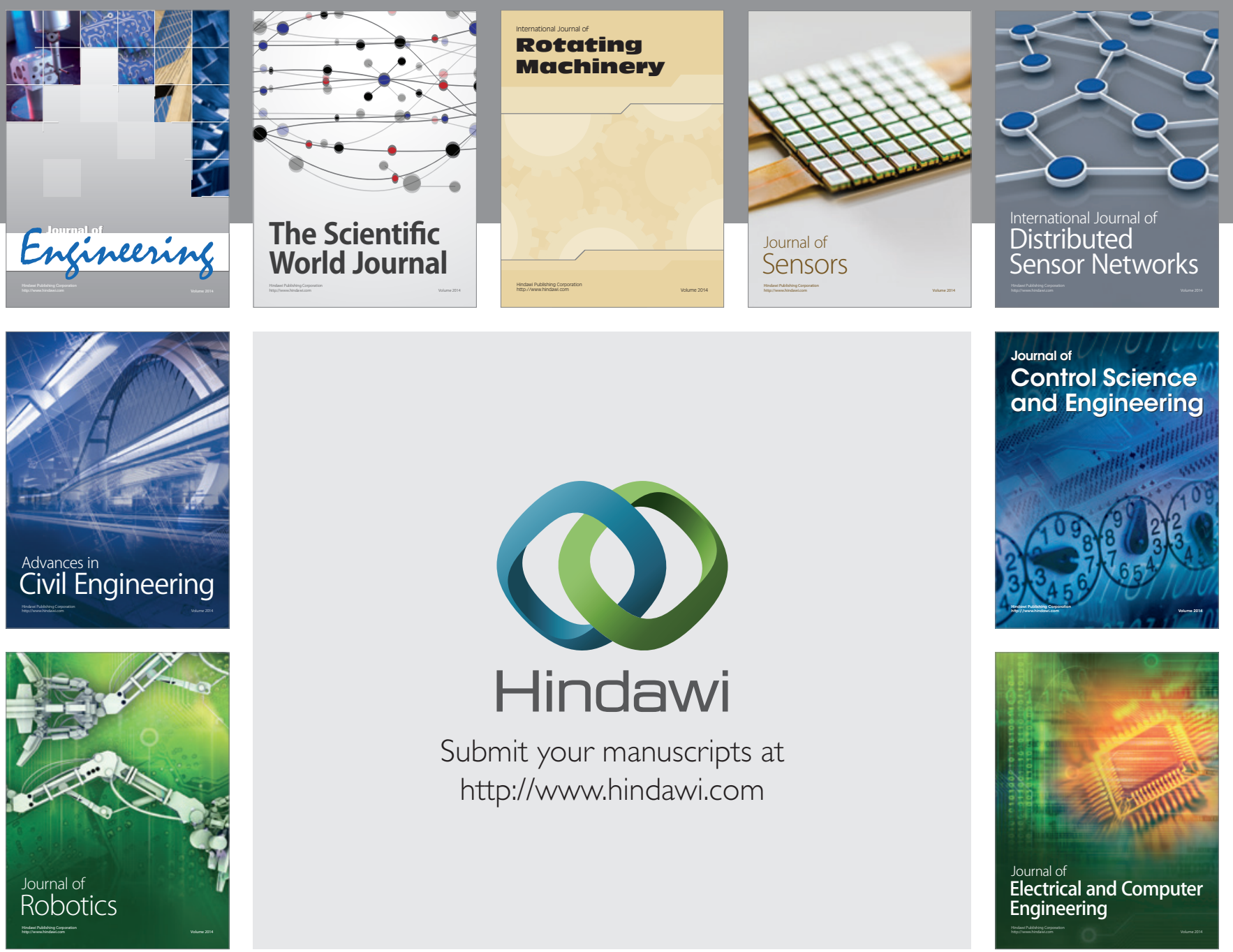

Submit your manuscripts at

http://www.hindawi.com
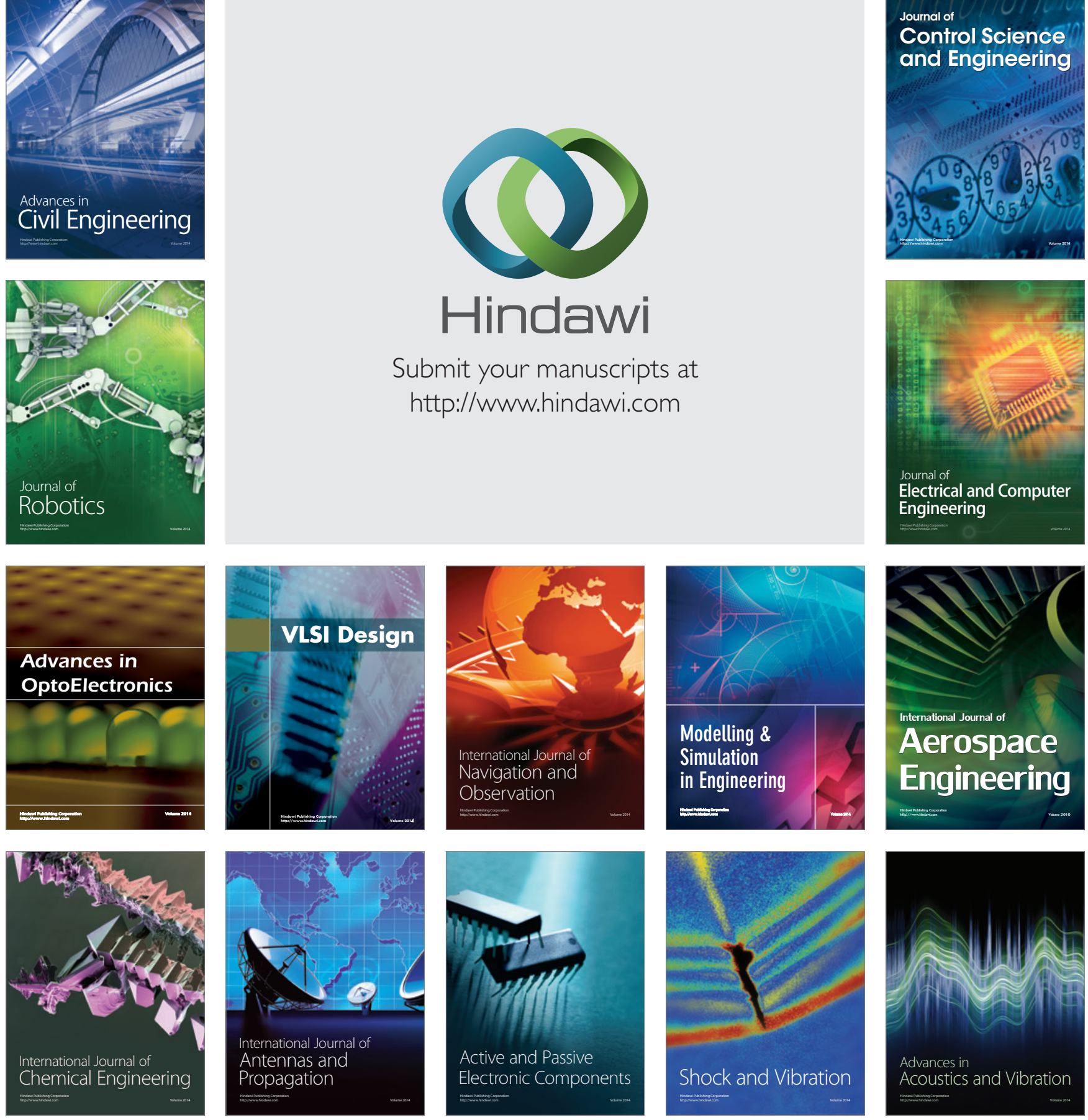